\title{
Analysis on the Design Theory and Design Method of Interactive Landscape
}

\author{
Kong Qingjun \\ College of Fine Arts, Hubei Normal University, Huangshi, Hubei 435002, China
}

Keywords: Interactive Landscape; Design Theory; Design Methods; Layer

\begin{abstract}
This paper focused on the theory and method of interactive landscape design. By carefully interpreting the different layers of interactive landscape design, the author explored the organizational form and design process of interactive landscape design, and also comprehensively summarized and elaborated the circular design process of the interactive life cycle of landscape design. Then, scientific design proposals were presented based on the different layers of interactive landscape design.
\end{abstract}

\section{Introduction}

Under the influence of the information age, the lifestyle of people has undergone significant changes. The concrete manifestation is that people's perception of the external environment tends to be superficial. They pay more attention to the diversified use of landscapes and the spatial form of landscapes is characterized by specialization. In the era when diversified information is widely used, the emergence of many derivative technologies has made modern design have new ideas, and various fields have begun to use interactive design extensively.

\section{The application status of interactive landscape design}

\section{Proposal of design concept}

The concept of interactive design was first proposed by the founder of IDEO and it has caused a lively discussion around the world. The so-called interaction design focuses on analyzing from the users' viewpoint, clarifying their visual experience and making them feel happy with new technologies. The ultimate goal of this type of design is to meet users' need for usability and experience, and thereby promote the steady improvement of their use of the related products [1]. Interactive design itself is an effective fusion of multiple disciplines, so it has a wide range of applications and has achieved remarkable results in product design research and practice. Interactive design has also led to the development of computer technology, virtual reality technology and other technologies, so that it shows value in a variety of fields. Today, with the steady development of science and technology, the concept of interactive design has gradually penetrated into all aspects of our life and gradually evolved toward diversification.

2. Application of landscape design

At the current stage, landscape design mainly uses a more appropriate way to meet a variety of needs. For example, the outdoor display space pays more attention to the perception and behavior of visitors, while the interactive art installation focuses on the interactive design method in the landscape. Interaction design also has relatively typical cases in the practice of landscape design. Here takes the Crown Fountain in Chicago's Millennium Park as an example. The fountain is composed of a black granite reflecting pool placed between a pair of glass brick towers. The towers are 50 feet $(15.2 \mathrm{~m})$ tall and they use light-emitting diodes (LEDs) to display digital videos on their inward faces, thus attracting many foreign tourists. The water operates from May to October, intermittently cascading down the two towers and spouting through a nozzle on each tower's front face, which makes the visitors feel refreshed. This fountain shows new meaning by visualizing the original static things. 


\section{Theoretical study of interactive landscape design}

\section{Interactive landscape}

The so-called interactive landscape refers to the landscape with interactive attributes. The design space and various elements of this kind of landscape show the phenomenon of two-way communication, mutually influencing each other. The interaction focus here refers to the information transmission between the landscape and people, between people and people and between self-reflections. The information medium is the combination of objective material and subjective consciousness. Interaction behaviors often involve interactive information which is the interaction medium. In the process of interaction, the two-way communication of information is carried out and the sending and receiving of information is completed in this process. The medium of interaction will change if there are significant differences in the interacting objects. From the perspective of the participants, the output information contains explicit information and implicit information. Explicit information concludes many aspects such as behavior and language; these kinds of information can be directly interpreted and directly interacted with the landscape [2]. The implicit information also contains many aspects like feelings and cognition; and the interaction of these kinds of information is often completed by properly absorbing the information. In the perspective of the landscape, the output information can be appropriately distinguished as two important types, namely the physical image and perceived image. The physical image contains the static scene and dynamic scene. The static scene mainly refers to the landscape that can be recognized by the naked eye. The latter is the dynamic landscape, such as the movement of objects, the change of an image, and so on.

2. Interactive landscape design

Interactive landscape design mainly refers to the whole process in which designers appropriately create interactive landscape by using basic ideas of interactive design. The focus of this kind of design is to regard the participants' needs as an important direction. Designers use the means of enhancing the output information of landscape to appropriately feedback the information sent by the participants, thereby achieving optimization of the landscape information cycle.

\section{Analytic hierarchy}

The design of the interactive landscape contains three very important parts: participants, designers and landscapes. They are all important aspects to achieve the circulation, feedback and delivery of information. However, since the transmission of information itself is instantaneous and also time-delay, the layer of interactive landscape cannot be divided in isolation. In other words, it is needed to conduct reasonable analysis of the current things and judge the overall development trajectory of things in the long run.

(1). The perceptual interaction layer

The focus of the perceived environment is that people get the basic information of the environment according to their feelings and then make corresponding psychological reactions. In the perceptual layer, the needs of users are often stuck to the surface things. The corresponding requirements of this kind are focused on the recognition of the things themselves and the sensory stimulation. In terms of the actual experience of users, it is necessary to analyze the impact of demand goals. The designer needs to start from a comprehensive understanding of the visitors' in the design stage and use a variety of means to enable them to better gain new insights. The result of perceptual interaction feedback focus on gaining a new understanding in interactive landscape and individual experiences, and a better model of perceptual interaction can be constructed by integrating the interaction of various elements.

(2). Behavioral interaction layer

The focus of environmental behavior research is to judge the classification of people's own behavior patterns in the corresponding landscape space and the corresponding trend that the two models show. The behavioral interactions in the landscape can be divided into various types such as the individual interaction behavior and necessary group interaction behavior; the designers can actively follow the basic rules and implement the scientific design scheme [3]. 
(3). Emotional interaction layer

Emotional resonance generally needs to be based on a certain behavioral awareness and behavioral experience. Only in this way can the basic needs be met. Emotional interaction can be appropriately divided into different categories according to the subject and object in the process of interacting. Expected emotional focus refers to the expectation of users for the landscape itself, which involves cultural, intellectual and other factors. The design should actively control the theme on the basis of the emotional goals. The level of interaction is mainly determined by the imagination space of the theme setting and also relates to the quality of the participants themselves. The real-time emotional interaction is often the emotional resonance between the subject and object in the landscape space when the theme has been determined, so it can highlight the obvious instantaneous characteristics. The design in this stage needs to actively create a good situation for the participants so that the space atmosphere can be rendered. This will make landscape participation no longer simply a behavior activity, but a process of blending thoughts and emotions in landscape narration.

\section{The organization form of interactive landscape design}

At the stage of interactive landscape design, it is often necessary to show the feature of obvious multi objects and multi layers. Therefore, participants show a very important position in the specific design process, which also reflects the diversity of the organizational forms of interactive landscape design.

\section{Designer model}

This pattern is consistent with the organization form of traditional landscape design, which is mainly based on the designer's on-site investigation to achieve a more reasonable analysis of the investigation results [4]. This form advocates that designers should pay attention to users' living needs in a timely manner, and at the same time, they are expected to have the most basic foresight. In real life, designers sometimes deviate from the original goal at the design stage, so it is difficult to plan scientifically according to their subjective will.

\section{User evaluation model}

This mode is mainly aimed at the landscape built according to the designer mode. It can timely analyze the experience of users and properly adjust the landscape plan with the help of POE method. One of the most critical problems is that there are significant differences between participants' expected demand goals and specific plans in the early design stage. Therefore, the implementation of the evaluation model generally results in high reconstruction costs and serious resources waste.

3. Public participation design model

This mode is mainly completed by designers and participants. Therefore the design ideas involved are a collection of various ideas. It can improve the design effect better, and at the same time, it can set more definite goals and ensure the realization of the interests of all parties. Before determining the design plan, the designers must take effective control from the perspective of professionals and predict the actual needs and social factors, so as to fully interpret visitors' specific opinions in the interactive landscape and ensure that possible changes will be considered in due course.

\section{Method analysis of interactive landscape design}

1. Process of interactive landscape design

The focus of interactive landscape design is to put people's needs in a relatively important position, so the whole process is greatly systematic. The investigation and analysis of the current situation and the determination of design objectives are all very important [5]. In the process of design, enough attention should be given to the reasonable feedback of the content in the subsequent stage. Only by solving the detailed problems one by one can designers have reasonable changes in the standpoint of understanding the problems, comprehensively interpret the contents that have been ignored in the past, raise reasonable doubts about the initial evaluation, properly 
determine the corresponding targets and ensure a better circulation of the interactive landscape design process. The rules of interactive landscape design belong to the circular design process of the whole life cycle. If it can be properly distinguished from the previous linear process, this will terminate the acceptance of the project itself.

2. Countermeasures of interactive landscape design

(1). The perceptual interaction layer

The design for the layer is very important. It focuses on the initial impression of the landscape as well as the shape and color of the landscape itself. Because people's sensory system is divided into many aspects, there will be significant differences in physical and mental perception when they are subjected to different stimuli. In the specific process of design, if the landscape can be used to give reasonable stimulation according to the sensory characteristics, it can fully attract visitors' attention and make them have better psychological perception [6].

(2). Behavioral interaction layer

At the behavioral interaction layer, what should be emphatically analyzed is the degree of people's participation in the landscape. At the same time, the usability of landscape functions and the existing behavioral relationship between individuals and groups are also aspects that need to be taken into account. The key point of the design is to appropriately enrich the landscape interaction modes so as to ensure the steady improvement of users' pleasure and promote the occurrence of repeated experience. According to the above basic needs, attention should be given to the availability of landscape space to create a good landscape space, so as to better cater to people's behavior habits. Designers can appropriately create more opportunities with the help of different landscape space forms, arrange more functional flexible activity spaces and use various means to promote human-landscape interaction, so as to ensure close communication among various parties. It is also feasible to use digital means to highlight the interest of landscape facilities and gradually break the barrier of human-landscape communication, with the purpose of improving people's participation and create more suitable space.

(3). Emotional interaction layer

From the perspective of expected emotional interaction, the focus of the design needs to be placed on the formulation of landscape themes, and at the same time it needs to focus on the image control of the overall environment. Designers can enrich various connotations by means of design metaphors, so that participants can realize proper emotional interaction [7]. If the image of the landscape can be presented before the visitors touch it, they will be able to get emotional recognition in time. The design focus of real-time emotional interaction is to create a high-quality environment for participants, to ensure that the atmosphere of the space is reasonably created, and to properly create the landscape environment scene feeling to the fullest, so that the distance between users and the landscape environment is properly narrowed, thus demonstrating the value of landscape existence.

\section{Conclusions}

Interactive landscape design should keep in line with the development of the times while paying attention to the basic needs of users. In the process of practice, designers should actively deal with complex and diverse problems to ensure that the design of landscape environment is in place and reasonable. At present, people put forward higher requirements for the landscape environment and advocate the application of new design theories and methods. Interactive landscape design regards the relationship between human and landscape as a top priority, ensuring that landscape design can gradually return and improve towards the direction of humanized design, and thus it caters to people's yearning for a better life. 


\section{References}

[1] Lu Xiaocui. Demand and Application of Virtual Reality Technology in Modern Environmental Art Design [J]. Electronics World, 2018 (17): 194+196.

[2] Jia Hongxu, Wang Yulong. “Digital Media Garden Design” -- Innovative Application Research in the Waiting Hall of High-speed Railway Stations in Dalian [J]. Beauty \& Times (Urban Edition), 2018 (01): 40-41.

[3] Qu Siyi, Wang Wei, et al. Discussion on the Greening Landscape Design Based on Interactive Urban Road Nodes -- Taking the Road Nodes of Government Roads in Wenjiang District of Chengdu as an Example [J]. Jilin Forestry Science and Technology<JiLin LinYe KeJi>, 2018, 47 (01): 33-35+38.

[4] Ding Mingjing. Linear Recreation Space Optimization Based on Landscape Interactive Design Concept -- Taking the Tour Path Design of Sanshi Gang Wetland Protection Project in Hefei as an Example [J]. Journal of Chongqing Technology and Business (Natural Sciences Edition), 2016, 33(02): 115-119.

[5] Pan Li. The Concept, Method and Technology Reconstruction of Landscape Planning from the Perspective of Ecological Wisdom [J]. Anhui Agricultural Sciences, 2015, 43(35): 258-261.

[6] Hu Haihui, Chen Xu, Gong Shufang. Research on the Application of Virtual Reality Technology in the Teaching of "Landscape Design of Landscape Plants” [J]. Journal of Heilongjiang College of Education, 2015, 34 (03): 64-66.

[7] He Yali. Landscape Design of Urban Entrance with Diversity, Ecology and Interaction [J]. Anhui Architecture, 2013, 20 (03): 17-18+125. 